\title{
DISTRIBUTED COOPERATIVE CONTROL OF MULTIPLE VEHICLE FORMATIONS USING STRUCTURAL POTENTIAL FUNCTIONS 1
}

\author{
Reza Olfati-Saber Richard M. Murray
}

\author{
Control and Dynamical Systems \\ California Institute of Technology \\ Pasadena, CA 91125 \\ \{olfati,murray\}@cds.caltech.edu
}

\begin{abstract}
In this paper, we propose a framework for formation stabilization of multiple autonomous vehicles in a distributed fashion. Each vehicle is assumed to have simple dynamics, i.e. a double-integrator, with a directed (or an undirected) information flow over the formation graph of the vehicles. Our goal is to find a distributed control law (with an efficient computational cost) for each vehicle that makes use of limited information regarding the state of other vehicles. Here, the key idea in formation stabilization is the use of natural potential functions obtained from structural constraints of a desired formation in a way that leads to a collision-free, distributed, and bounded state feedback law for each vehicle.
\end{abstract}

Keywords: Distributed Control, Autonomous Vehicles, Multivehicle Systems, Graph Theory, Formation Stabilization, Potential Functions

\section{INTRODUCTION}

Control of systems consisting of multiple vehicles (or agents) with several sensors and actuators that are intended to perform a coordinated task is currently an important and challenging field of research. This is due to broad range of applications of multi-agent systems in automated highways, air traffic control, control of a cluster of telescopes, satellite formations, coordinated control of vehicles involved in search and rescue operations, cooperative control of mobile robots capable of playing games (e.g. soccer), and formation flying of autonomous unmanned aerial vehicles (UAV).

In this paper, our goal is to address distributed structural stabilization of formations of multiple vehicles using structural potential functions ob-

\footnotetext{
1 This work was supported by DARPA under contract F33615-98-C-3613.
}

tained naturally from the formation graphs of the vehicles. An alternative is to use artificial potential functions as in Leonard and Fiorelli (2001).

The use of the second approach - imposing artificial potentials among different agents - in robotics applications began near a decade ago, e.g. see Rimon and Koditschek (1992). More recently, in Leonard and Fiorelli (2001), artificial potential functions have been used for coordination of multiple vehicles. The main feature of such potential functions is that they are constant beyond a certain distance $d>0$. This leads to a distributed control law for each vehicle which does not depend on the position and velocity (i.e. state) of all other vehicles in the formation. However, the distributed controller suggested in Leonard and Fiorelli (2001) does not guarantee stabilization of the system of multiple vehicles to a unique desired formation. According to Leonard and Fiorelli (2001), depending on the number of vehicles and 
their initial conditions, the formation of the system of vehicles might converge to one of the many possible formations which is rather undesirable. This nonuniqueness effect occurs whenever ambiguity of a formation graph is not taken into account.

In our work, the distributed property of the controller of each vehicle is achieved by imposing a specific structure on a weighted graph associated with the formation of the vehicles. In other words, the interconnections of the formation graph of the vehicles are designed in such a way that guarantees collision-free stabilization of the system of multiple vehicles to a unique and unambiguous desired formation.

In Fax and Murray (2001), the Laplacian of a formation graph is used to determine a Nyquisttype criterion for stability analysis of multi-vehicle formations in closed-loop with linear controllers. Though the control design approach in this work is substantially different from the methods and tools used in Fax and Murray (2001), it turns out that in both works, the information flow among the vehicles, modeled as a graph, has an important role in stability (or lack of stability) of the closedloop formation dynamics.

Formation tracking and reorientation problems are topics that are addressed in an upcoming sequel of this paper.

Here is an outline of the paper: In section 2, we define basic notions related to graph theory, representation of multi-agent formations, structural potential functions, and formation stabilization. Our main results are presented in section 3. Finally, in section 4 we make concluding remarks.

\section{BASIC NOTIONS AND DEFINITIONS}

In this section, we introduce the notion of metric formation graphs for coordination of multiple vehicles. We begin by explaining the basics of graph theory and later introduce a class of graphs that are more convenient for dealing with control of systems consisting of multiple agents (or vehicles). The reader should refer to Diestel (1997) for more details on graph theory.

The planar formation graph (or network) of multiple vehicles is a planar directed graph or a triplet $\mathcal{G}=(\mathcal{V}, \mathcal{A}, \mathcal{E})$ that consists of a set of vertices $\mathcal{V}$ with elements $v_{i}$ and $i \in \mathcal{I}=\{1,2, \ldots, n\}(\mathcal{I}$ is called an index set), an adjacency matrix $\mathcal{A}$ with elements $a_{i j}$ in $\{0,1\}$, and a set of edges $\mathcal{E}$ with elements $e_{i j}=\left(v_{i}, v_{j}\right) \in \mathcal{V} \times \mathcal{V}$ for $i, j \in \mathcal{I}$. We say $v_{j}$ is a neighbor of $v_{i}$ if $e_{i j} \in \mathcal{E}$. A graph $\mathcal{G}$ is called undirected if it satisfies the property $\forall i, j \in \mathcal{I}, i \neq j: e_{i j} \in \mathcal{E} \Leftrightarrow e_{j i} \in \mathcal{E}$.
From a computational complexity point of view, for systems consisting of multiple agents (or vehicles), both the complexity of control design (e.g. the dimension of the space in which a given convex optimization problem has to be solved) and the computational cost of implementing the obtained controller for each vehicle is proportional to the degree in which a network of agents are interconnected.

Let us define the connectivity number of vertex $v_{i}$ as the number of edges leaving $v_{i}$ and denote it by $\left|v_{i}\right|$. Then, we define the maximum connectivity of $\mathcal{G}$ as $m=|\mathcal{V}|:=\max _{i \in \mathcal{I}}\left|v_{i}\right|$ and the connectivity ratio of $\mathcal{G}$ as

$$
\rho(\mathcal{G})=\frac{|\mathcal{V}|}{|\mathcal{I}|}
$$

Notice that by definition, $\rho(\mathcal{G}) \in[1 / n,(n-$ $1) / n] \subset(0,1)$ where $n=|\mathcal{I}|$. In addition, $\rho(\mathcal{G})=$ $(n-1) / n$ corresponds to a graph in which each vertex is connected to every other vertex excluding itself and $\rho(\mathcal{G})=1 / n$ corresponds to a graph that each vertex is connected to at most one more vertex. We refer to graphs with $\rho(G) \ll 1$ as weakly connected graphs. Also, graphs with $\rho(\mathcal{G})=1-\epsilon$ such that $0<\epsilon \ll 1$ are referred to as strongly connected graphs.

For instance, consider a directed binary tree $\mathcal{G}(l)$ with $l \geq 1$ levels and $n=2^{l}-1$ nodes. For $\mathcal{G}(l)$, $m=|\mathcal{V}|=2$ is fixed and as the number levels $l$ increases, the connectivity ratio $\rho(\mathcal{G}(l))=2 /\left(2^{l}-\right.$ 1) exponentially decreases as a function of $l$. In other words, a binary tree with relatively large number of levels $(l \gg 1)$ is an example of a weakly connected graph.

In this paper, we are interested in formation graphs of multiple vehicles that are weakly connected. Therefore, the adjacency matrices $\mathcal{A}$ of the cases of interest are sparse matrices. This motivates us to use a compact representation of $\mathcal{A}$ that is more appropriate for weakly connected graphs. For this purpose, instead of $\mathcal{A}$, we use the connectivity matrix $\mathcal{C}$ which is an $n \times m$ matrix where $m=|\mathcal{V}|$ (thus, $\rho(\mathcal{G})=m / n$ ). Let $c_{i j} \in \mathcal{V}_{e}$ denote an element of $\mathcal{C}$, where $\mathcal{V}_{e}=\mathcal{V} \cup$ $\left\{v_{\infty}\right\}, v_{\infty} \notin \mathcal{V}$ is the extended set of vertices with an infinite vertex $v_{\infty}$. If all vertices have equal number of neighbors, then define $\mathcal{V}_{e}=\mathcal{V}$. In addition, let $\mathcal{N}_{i}$ be the set of neighbors of the vertex $i$, i.e. $\mathcal{N}_{i}:=\left\{v_{j} \in \mathcal{V}: e_{i j} \in \mathcal{E}\right\}$ and $\mathcal{K}_{i}:=\left(k_{1}, k_{2}, \ldots, k_{n_{i}}\right) \in \mathcal{I} \times \mathcal{I} \times \cdots \times \mathcal{I}$ with $n_{i}=\left|v_{i}\right|$ be an ordered $n_{i}$-tuple (i.e. for all $s<t$, $k_{s}<k_{t}$ ) of the indices of the elements of $\mathcal{N}_{i}$. Then, we define $c_{i j}$ as follows:

$$
c_{i j}:=\left\{\begin{array}{l}
v_{k_{j}}, \text { if } 1 \leq j \leq\left|v_{i}\right| \\
v_{\infty}, \text { otherwise }
\end{array}\right.
$$

i.e. the first $n_{i}=\left|v_{i}\right|$ elements of the $i$ th row of $\mathcal{C}$ are equal to the neighbors of $v_{i}$ which are ordered increasingly according to their vertices. The 
remaining elements are all equal to the infinity vertex $v_{\infty}$ (and are essentially unimportant).

Denote the coordinates of $v_{i}$ in $\mathbb{R}^{2}$ by $q_{i}$. We define a metric formation graph $(M F G)$ as a triplet $\mathcal{G}:=$ $\left(\mathcal{V}_{e}, \mathcal{C}, \mathcal{D}\right)$ where $\mathcal{D}$ is called the distance matrix and has the same dimension as the connectivity matrix $\mathcal{C}$. The element $d_{i j}$ of $\mathcal{D}$ is the distance between $v_{i}$ and its $j$ th neighbor $v_{k_{j}}$ if $1 \leq j \leq\left|v_{i}\right|$ and infinity otherwise, i.e.

$$
d_{i j}:= \begin{cases}\left\|q_{k_{j}}-q_{i}\right\|, & \text { if } 1 \leq j \leq\left|v_{i}\right| \\ \infty, & \text { otherwise }\end{cases}
$$

where $\|\cdot\|$ denotes the 2-norm.

Example 1. Consider the metric formation graphs $\mathcal{G}_{a}$ and $\mathcal{G}_{b}$ as shown in Fig. 1 (a) and (b), respectively. The connectivity ratios for these two graphs are $\rho\left(\mathcal{G}_{a}\right)=2 / 4$ and $\rho\left(\mathcal{G}_{b}\right)=3 / 4$ which means $\mathcal{G}_{b}$ is more connected than $\mathcal{G}_{a}$. The set of vertices, connectivity matrix, and the distance matrix for $\mathcal{G}_{a}$ and $\mathcal{G}_{b}$ are given in the following

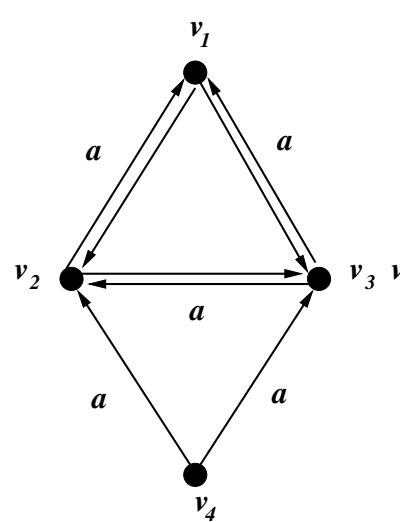

(a)

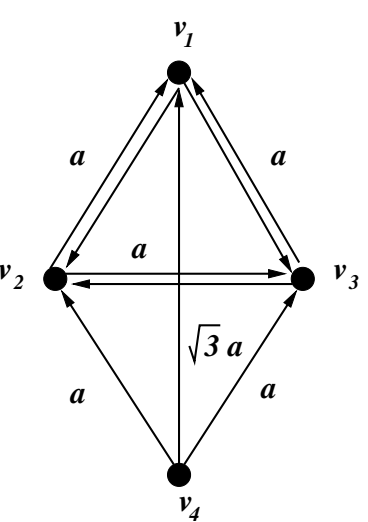

(b)
Fig. 1. Two directed metric formation graphs each with four vertices.

$$
\begin{gathered}
\mathcal{V}_{e}^{(a)}=\left\{v_{1}, v_{2}, v_{3}, v_{4}\right\}, \mathcal{V}_{e}^{(b)}=\left\{v_{1}, v_{2}, v_{3}, v_{4}, v_{\infty}\right\} \\
\mathcal{C}^{(a)}=\left[\begin{array}{ll}
v_{2} & v_{3} \\
v_{1} & v_{3} \\
v_{1} & v_{2} \\
v_{2} & v_{3}
\end{array}\right], \mathcal{C}^{(b)}=\left[\begin{array}{lll}
v_{2} & v_{3} & v_{\infty} \\
v_{1} & v_{3} & v_{\infty} \\
v_{1} & v_{2} & v_{\infty} \\
v_{1} & v_{2} & v_{3}
\end{array}\right] \\
\mathcal{D}^{(a)}=\left[\begin{array}{ll}
a & a \\
a & a \\
a & a \\
a & a
\end{array}\right], \mathcal{D}^{(b)}=\left[\begin{array}{ccc}
a & a & \infty \\
a & a & \infty \\
a & a & \infty \\
\sqrt{3} a & a & a
\end{array}\right]
\end{gathered}
$$

One notices that the first and last row of $\mathcal{C}^{(a)}, \mathcal{D}^{(a)}$ are exactly equal for $\mathcal{G}_{a}$, but the same rows in $\mathcal{C}^{(b)}, \mathcal{D}^{(b)}$ are substantially different for $\mathcal{G}_{b}$. In fact, if the vertices $v_{1}$ and $v_{4}$ coincide in Fig. 1 (a), then the $\mathcal{C}$ and $\mathcal{D}$ matrices corresponding to this graph remain the same as $\mathcal{C}^{(a)}$ and $\mathcal{D}^{(a)}$. In addition, in Fig. 1 (a), if the edges $\left(v_{2}, v_{3}\right)$ and $\left(v_{3}, v_{2}\right)$ are removed, the distance between $v_{2}$ and $v_{3}$ can take any arbitrary value in $[0,2 a]$ and the graph will not be uniquely determined by its $\mathcal{C}$ and $\mathcal{D}$ matrices. Both of these ambiguities are undesirable in local/global formation stabilization of multiple vehicles.

We make use of the following definitions in this work: A formation graph $\mathcal{G}$ with $n$ vertices is called rigid if it has at least $2 n-3$ critical links where any directed/undirected edge counts as a single link (i.e. the edges $(v, w),(w, v) \in$ $\mathcal{E}, v \neq w$ count as a single link). A link that eliminates a single flexible degree of freedom of the graph is called a critical link. According to this definition, by removing the link between $v_{2}$ and $v_{3}$ in Fig. 1 (a), one gets a nonrigid formation graph. If there exists a pair of distinct vertices $v, w \in \mathcal{V}$ $(v \neq w)$ in $\mathcal{G}$ that share the same neighbors which are located on a straight line not passing through $v, w$, then $\mathcal{G}$ is called a foldable graph and $[v, w]$ is called a foldable pair. In addition, a formation graph $\mathcal{G}$ with no foldable pairs is called an unfoldable graph. A formation graph $\mathcal{G}$ that is both rigid and unfoldable is called unambiguous (i.e. can be uniquely constructed from its $\mathcal{C}$ and $\mathcal{D}$ matrices). In other words, any nonrigid or foldable graph is ambiguous. For planar formation graphs, a foldable pair of vertices $[v, w] \in \mathcal{V} \times \mathcal{V}$ can be rendered unfoldable by adding a new edge $(v, w)$ to $\mathcal{E}$, e.g. adding the edge $\left(v_{4}, v_{1}\right)$ to $\mathcal{G}_{a}$ in Fig. 1 (a) renders the graph unfoldable and therefore unambiguous.

Denote the set of $s$ (non-redundant) constraints of the lengths of the edges in a metric formation graph $\mathcal{G}$ by $\phi_{l}\left(q_{i}, q_{j}\right)=0$ for $l=1, \ldots, s$ and $i, j \in \mathcal{I}, i \neq j$. Each element $\phi_{l}\left(q_{i}, q_{j}\right)$ is a function of $\left(q_{i}, q_{j}\right)$, the coordinates of $v_{i}, v_{j}$, as the following

$$
\phi_{l}\left(q_{i}, q_{j}\right):=\left\|q_{i}-q_{j}\right\|-d_{i j}, \quad e_{l}:=\left(v_{i}, v_{j}\right) \in \mathcal{E}
$$

where $d_{i j}$ is an element of the distance matrix $\mathcal{D}$ corresponding to the length of the edge $\left(v_{i}, v_{j}\right)$. We refer to the set of equations in (4) as the set of algebraic structural constraints of $\mathcal{G}$. Denoting $q=\operatorname{col}\left(q_{1}, \ldots, q_{n}\right)$ and $\Phi(q):=\operatorname{col}\left\{\phi_{l}\right\}_{l=1}^{s}$, the set of structural constraints of $\mathcal{G}$ can be expressed as $\Phi(q)=0$. We refer to $\Phi(q)$ as the structural constraint function of the formation graph $\mathcal{G}$. Let $\mathcal{G}$ be a metric formation graph with structural constraint function $\Phi(q)$. A point $\bar{q} \in \mathbb{R}^{2 n}$ is called an equilibrium formation iff $\Phi(\bar{q})=0$. Equivalently, we say that the formation $\mathcal{G}$ is centered at $\bar{q}$. It is easy to check that algebraic structural constraints of a metric formation graph remain invariant under rotation and translation of all the coordinates, i.e. Let $\hat{q}_{i}=R q_{i}+b, \hat{q}_{j}=R q_{j}+$ $b$ where $(R, b) \in S E(2)$ and $R^{T} R=I$, then

$$
\left\|\hat{q}_{j}-\hat{q}_{i}\right\|=\left\|R\left(q_{j}-q_{i}\right)\right\|=\left\|q_{j}-q_{i}\right\|
$$

Therefore, by uniqueness of an equilibrium formation $\bar{q}$, we mean uniqueness up to an $S E(2)$ sym- 
metry. For a system of multiple vehicles, we refer to a positive definite function $V(q)$ that depends on the set of algebraic structural constraints of the formation graph of the vehicles as a structural potential function. A special class of such potential functions is $V(q):=\langle\Phi(q), Q \Phi(q)\rangle$. We call this the natural potential function of $\mathcal{G}$ where $\langle\cdot, \cdot\rangle$ denotes the standard inner product in $\mathbb{R}^{s}$.

In this paper, our main goal is to address structural formation stabilization of a group of $n$ vehicles to a desired undirected metric formation graph using structural potential functions (e.g. a natural potential function $V(q)$ with $Q=I)$. Consider a planar formation of $n$ vehicles with the following dynamics

$$
\left\{\begin{array}{l}
\dot{q}_{i}=p_{i} \\
\dot{p}_{i}=u_{i}
\end{array}\right.
$$

where $q_{i}, p_{i}, u_{i} \in \mathbb{R}^{2}$ and $i=1,2, \ldots, n$. The set of equations in (5) can be rewritten in a more compact way as a multi-variable double-integrator

$$
\left\{\begin{array}{l}
\dot{q}=p \\
\dot{p}=u
\end{array}\right.
$$

with $q, p, u \in \mathbb{R}^{2 n}$. Now, we are ready to define a stabilization problem for the formation of these multiple vehicles.

Definition 1. (structural formation stabilization) Let $\mathcal{G}$ be a metric formation graph of $n$ vehicles with the overall dynamics given in (6) centered at the equilibrium formation $\bar{q} \in \mathbb{R}^{2 n}$. Denote the equivalent class of desired equilibrium points of (6) satisfying $\Phi(q)=0$ up to a symmetry by $[\bar{x}]=$ $\operatorname{col}([\bar{q}], 0) \in \mathbb{R}^{4 n}$. Then, by structural formation stabilization of a group of $n$ vehicles to $\mathcal{G}$, we mean (global/local/asymptotic) stabilization of system (6) to the equilibrium formation manifold $[\bar{x}]$ in the sense of Lyapunov.

Definition 2. (neighborhood of a formation) Let $\mathcal{G}=\left(\mathcal{V}_{e}, \mathcal{C}, \mathcal{D}\right)$ be a metric formation graph centered at $\bar{q} \in \mathbb{R}^{2 n}$. Let $V(q)$ be a positive definite structural potential function for $\mathcal{G}$ satisfying $V(\bar{q})=0$. Denote a connected compact neighborhood of the point $\bar{q}_{i} \in \mathbb{R}^{2}$ by $\Omega_{i} \subset \mathbb{R}^{2}$ and let $c \geq 0$ be a nonnegative constant. We define a c-level neighborhood of $\mathcal{G}$ induced by $V(q)$ as $\Omega(c)=\bigcup_{i \in \mathcal{I}} \Omega_{i} \subset \mathbb{R}^{2}$ such that the following conditions hold:

i) The $\Omega_{i}$ 's are mutually disjoint, i.e. $\Omega_{i} \cap \Omega_{j}=$ $\emptyset, \forall i, j \in \mathcal{I}, i \neq j$.

ii) $V(q) \leq c, \forall\left(q_{1}, \ldots, q_{n}\right) \in \Omega_{1} \times \cdots \times \Omega_{n}$.

We call $c$ a separable level-value of $\mathcal{G}$ w.r.t. $V(q)$ iff for every choice of the set of neighborhoods $\Omega_{1}, \ldots, \Omega_{n}$ satisfying condition ii) of definition 2 , the $\Omega_{i}$ 's remain mutually disjoint.
Definition 3. (collision-free trajectories) Let $q_{i}(\cdot)$ with $i \in \mathcal{I}$ denote the trajectory of the $i$ th vehicle in a formation of $n$ vehicles. We say the position trajectories of the formation is collision-free iff the following property holds for

$$
\nexists t_{c} \geq 0: q_{i}\left(t_{c}\right)=q_{j}\left(t_{c}\right)
$$

for all $i, j \in \mathcal{I}, i \neq j$.

\section{MAIN RESULTS}

Let us view the formation of $n$ vehicles with a metric formation graph $\mathcal{G}$ and a structural constraints function $\Phi(q)$ centered at a desired equilibrium formation $\bar{q} \in \mathbb{R}^{2 n}$ as a simple Lagrangian system consisting of $n$ particles each with a constant mass $m_{i}>0$. We take the following natural potential function

$$
V(q):=\langle\Phi(q), \Phi(q)\rangle
$$

as the potential energy of the formation. Denoting the mass matrix by $M=\operatorname{diag}\left(m_{1}, \ldots, m_{n}\right)$, the structural Lagrangian function of the formation can be written as

$$
\mathcal{L}(q, \dot{q})=\frac{1}{2} \dot{q}^{T} M \dot{q}-\langle\Phi(q), \Phi(q)\rangle
$$

Due to symmetry of the structural constraints equations, $V(q)$ is invariant under rotation and translation of the elements of $\mathbb{R}^{2} \times \ldots \times \mathbb{R}^{2}$ (repeated $n$ times), and therefore $\mathcal{L}(q, \dot{q})$ is invariant under $S E(2)$ symmetry as well. Denoting the generalized momentum conjugate to $q$ by

$$
\pi=\frac{\partial \mathcal{L}}{\partial \dot{q}}=M \dot{q}
$$

the structural Hamiltonian of the system can be expressed as

$$
\mathcal{H}(q, \pi)=\frac{1}{2} \pi^{T} M^{-1} \pi+\langle\Phi(q), \Phi(q)\rangle
$$

For the special case of $M=I$ (i.e. $I$ is the identity matrix), we have $p=\pi$ and the structural Hamiltonian takes the following form

$$
\mathcal{H}(q, p)=\frac{1}{2}\|p\|^{2}+\langle\Phi(q), \Phi(q)\rangle
$$

Now, we are ready to present our main result on uniform local structural stabilization of formations of multiple vehicles.

Theorem 1. (local formation stabilization) Consider a group of $n$ identical vehicles with the formation dynamics given by

$$
\dot{q}=p, \dot{p}=u
$$

Then, the following smooth state feedback law

$$
u=K(q, p):=-\nabla\langle\Phi(q), \Phi(q)\rangle-D(q, \dot{q})
$$

with a damping force $D(q, \dot{q})$ that satisfies the following properties

$$
\langle D(q, p), p\rangle>0, \forall p \neq 0 \text { and } D(q, 0)=0
$$


guarantees collision-free local asymptotic stabilization of the vehicles formation to a desired undirected and unambiguous formation graph $\mathcal{G}$ centered at $\bar{q} \in \mathbb{R}^{2 n}$ for initial positions of vehicles starting in a neighborhood $\Omega(c)$ of $\mathcal{G}$ where $c>0$ is a separable level-value of $\mathcal{G}$ w.r.t. $V(q)$ and the initial velocities of vehicles belong to the set

$$
\Omega_{p}:=\left\{p \in \mathbb{R}^{2 n}:\|p\| \leq \sqrt{2 c^{2}-2\|\Phi(q(0))\|^{2}}\right\}
$$

In addition, the structural Hamiltonian $\mathcal{H}(q, p)$ in (11) is a valid local Lyapunov function for the system.

Proof. First, one observes that for all $p(0) \in \Omega_{p}$, the initial energy is bounded, i.e.

$$
\mathcal{H}(q(0), p(0)) \leq c^{2}
$$

In addition, due to $\dot{\mathcal{H}} \leq 0$ that follows from

$$
\begin{aligned}
\dot{\mathcal{H}} & =\langle\dot{p}, p\rangle+\langle\nabla V(q), p\rangle \\
& =\langle D(q, p), p\rangle \leq 0
\end{aligned}
$$

we have $\mathcal{H}(q(t), p(t)) \leq \mathcal{H}\left(q(0), p(0) \leq c^{2}, \forall t>0\right.$. Thus

$$
V(q(t)) \leq c^{2}, \quad \forall t \geq 0
$$

or

$$
\Phi(q(t)) \leq c, \quad \forall t \geq 0
$$

From definition 2, $q(t) \in \Omega(c)$ for all time $t>0$. Since $c \geq 0$ is a separable level-value, all the $\Omega_{i}$ 's are mutually disjoint (i.e. $\Omega_{i} \cap \Omega_{j}=\emptyset, \forall i \neq j$ ) and therefore the position trajectories of the $n$ vehicles remains collision-free (definition 3 ) for all time $t>0$ and never leave the neighborhood $\Omega(C)$ of $\bar{q}$. On the other hand, $\dot{\mathcal{H}}$ vanishes iff $p=0$. But the largest invariant set in $\Omega_{0}:=\left\{(q, p) \in \Omega(c) \times \Omega_{p}\right.$ : $p=0\}$ is the equilibrium formation $\{(\bar{q}, 0)\}$ and thus according to LaSalle's invariance principle $\{(\bar{q}, 0)\}$ is (locally) asymptotically stable for the system. Furthermore, $\mathcal{H}(q, p)$ is a valid Lyapunov function for the closed-loop system with a seminegative definite $\dot{\mathcal{H}}$.

Remark 1. Based on the definition of $\Omega_{p}$ (i.e. the set of initial velocities), the higher the separable level-value $c \geq 0$ of a local neighborhood of a formation equilibrium of $\mathcal{G}$, the larger the region of attraction for the control law in (13).

Remark 2. Since $\mathcal{H}(q, p)$ is a physically meaningful (local) control Lyapunov function for the formation dynamics, it is possible to perform robustness analysis for the obtained controller in (13) and test effects like string stability of the system.

Remark 3. The main drawback of the stabilization result in Theorem 1 is that it is only applicable to undirected graphs. In fact, the same problem exists in Leonard and Fiorelli (2001). This will be resolved in the sequel of this paper.
Theorem 2. (bounded feedback stabilization) Suppose all the conditions in Theorem 1 hold. Define the following structural potential function

$V_{b}=\frac{\bar{u} \lambda_{1}}{2} \sum_{i \in \mathcal{I}} \frac{1}{\left|J_{i}\right|} \sum_{j \in J_{i}}\left[1+\left(\left\|q_{i}-q_{j}\right\|-d_{i j}\right)^{2}\right]^{\frac{1}{2}}-1$ satisfying $V_{b}(\bar{q})=0$ where $\bar{q}$ denotes the coordinates of the non-infinity vertices of an unambiguous formation graph $\mathcal{G}$ and $J_{i}$ is the set of the indices of the neighbors of $v_{i} \in \mathcal{V}$. Then, the following smooth nonlinear state feedback

$$
u=-\nabla V_{b}(q)-\bar{u} \lambda_{2} \boldsymbol{\sigma}(p)
$$

gives a bounded distributed control law satisfying $\left\|u_{i}\right\| \leq \bar{u}$ which achieves collision-free local stabilization of the vehicles formation to $\mathcal{G}$ where $\boldsymbol{\sigma}: \mathbb{R}^{2 n} \rightarrow \mathbb{R}^{2 n}$ denotes a vector sigmoidal function with $n$ identical elements $\sigma_{2}(\cdot)$ defined by the $m$-dimensional sigmoidal function $\sigma_{m}(y)=$ $y / \sqrt{1+\|y\|^{2}}, y \in \mathbb{R}^{m}$. In addition, $\bar{u}>0$ and $\lambda_{1}, \lambda_{2} \in(0,1), \lambda_{1}+\lambda_{2}=1$.

Proof. Similar to the proof of Theorem $1, \mathcal{H}_{b}(q, p)$ defined as the following

$$
\mathcal{H}_{b}(q, p):=\frac{1}{2}\|p\|^{2}+V_{b}(q)
$$

is a valid Lyapunov function for the closed-loop system. Let $c>0$ be a separable level-value of $\mathcal{G}$ w.r.t. $V_{b}(q)$. Then, $\Omega:=\left\{(q, p) \in \mathbb{R}^{4 n}: \mathcal{H}_{b}(q, p) \leq\right.$ $c\}$ is an invariant set for the trajectories of the $n$ vehicles. In addition, the trajectories of all vehicles remain collision-free for all time $t>0$. To prove that the state feedback in (15) is uniformly bounded, let us express the control of the $i$ th vehicle $u_{i}$ explicitly as the following

$$
u_{i}=\sum_{j \in J_{i}} w_{i j} \boldsymbol{u}_{i j}-\bar{u} \lambda_{2} \sigma_{2}\left(p_{i}\right)
$$

where $w_{i j}$ is a scalar weight and $\boldsymbol{u}_{i j}$ is a unit vector defined as

$$
\begin{aligned}
w_{i j} & :=\frac{\bar{u} \lambda_{1}}{\left|J_{i}\right|} \sigma_{1}\left(\left\|q_{i}-q_{j}\right\|-d_{i j}\right) \\
\boldsymbol{u}_{i j} & :=\frac{q_{j}-q_{i}}{\left\|q_{j}-q_{i}\right\|}
\end{aligned}
$$

where $\sigma_{1}(y)=y / \sqrt{1+y^{2}}$ is a scalar bounded sigmoidal function. From the definition of $w_{i j}$, we have

$$
\sum_{j \in J_{i}}\left|w_{i j}\right|<\bar{u} \lambda_{1}
$$

Thus, the control law in (16) is a bounded distributed nonlinear state feedback satisfying the following bound

$$
\left\|u_{i}\right\| \leq \bar{u}\left(\lambda_{1}+\lambda_{2}\right)=\bar{u}
$$

where $u_{i}$ is explicitly given by

$u_{i}=\frac{\bar{u}}{\left|J_{i}\right|} \sum_{j \in J_{i}} \lambda_{1} \sigma_{1}\left(\left\|q_{i}-q_{j}\right\|-d_{i j}\right) \boldsymbol{u}_{i j}-\bar{u} \lambda_{2} \sigma_{2}\left(p_{i}\right)$ 
Fig. 2 demonstrates simulation results for distributed formation stabilization of 3 vehicles with bounded control to an equilateral triangle that each of its sides are of length $a=5$ using the controller given in (3) (see Theorem 2). The initial conditions and parameters are chosen as follows: $q(0)=(-10,14,-7,-9,20,20)^{T}, p(0)=0, \bar{u}=3$, and $\lambda_{1}=\lambda_{2}=0.5$. As another example, the simulation results for a formation of $n=6$ vehicles in shown in Fig. 3. The desired formation is depicted in Fig. 3 (b). The initial condition is $q(0)=$ $(-30,5,-20,-5,-10,7,0,-7,10,9,20,-9)^{T}$ and $p(0)=0$. Also, the control bound is $\bar{u}=10$ and $\lambda_{1}=\lambda_{2}=0.5$. From Fig. 3 (a), it is clear that no two vehicles collide including $v_{5}$ and $v_{6}$.

\section{CONCLUSION}

In this paper, we proposed a distributed control design approach for local collision-free stabilization of formations of multiple vehicles with undirected interconnection graphs using potential functions that are obtained naturally from the structural constraints of a desired formation. After minor modification of the potential function associated with the formation, we obtained a bounded stabilizing nonlinear state feedback for the formation with an arbitrary (small or large) upper bound. We provided two examples of structural formation stabilization of groups of $n=3$ and $n=6$ vehicles using distributed bounded state feedback laws. Global stabilization, tracking, and reorientation of formations of multivehicles with directed graphs using artificial potential functions is the topic of a forthcoming sequel of this paper.

\section{REFERENCES}

Diestel, R. (1997). Graph Theory. Vol. 173 of Graduate Texts in Mathematics. SpringerVerlag.

Fax, A. and R. M. Murray (2001). "Graph Laplacians and Vehicle Formation Stabilization". CDS Technical Report 01007. California Institute of Technology. http://www.cds.caltech.edu/ ${ }^{\sim}$ murray /papers/2001g_fm01-cds.html.

Leonard, N. E. and E. Fiorelli (2001). "Virtual leaders, artificial potentials, and coordinated control of groups". In: Proc. of the 40th IEEE Conference on Decision and Control. Orlando, FL.

Rimon, E. and D. E. Koditschek (1992). "Exact robot navigation using artificial potential functions". IEEE Trans. on Robotics and Automation pp. 501-518.

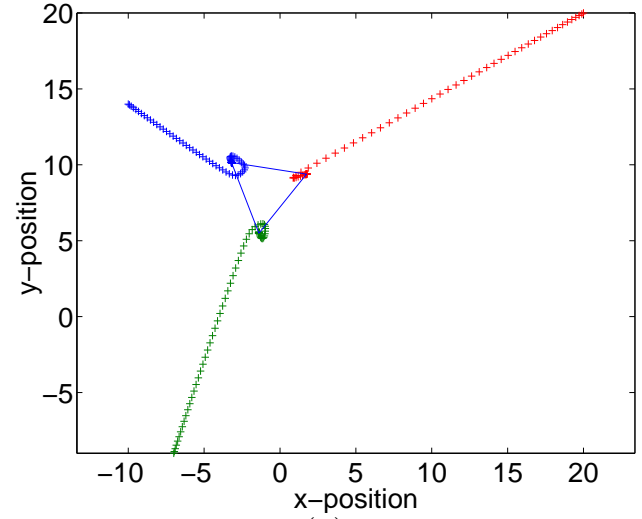

(a)

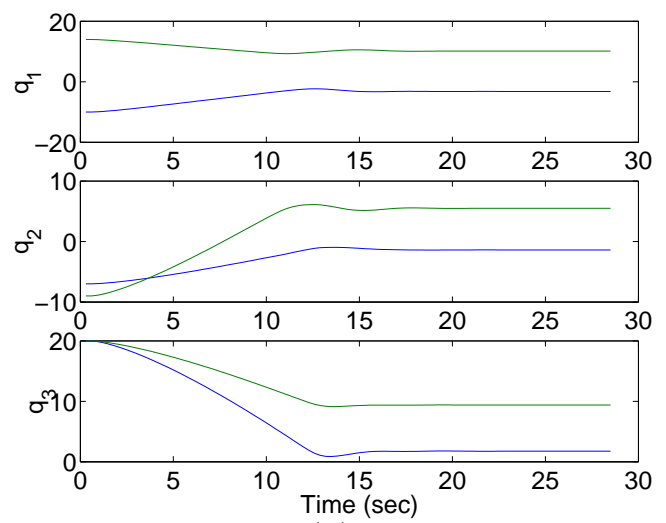

(b)

Fig. 2. Formation stabilization for 3 vehicles: (a) $q_{1}, q_{2}, q_{3}$ in $\mathbb{R}^{2}$ and (b) $q(\cdot)$.

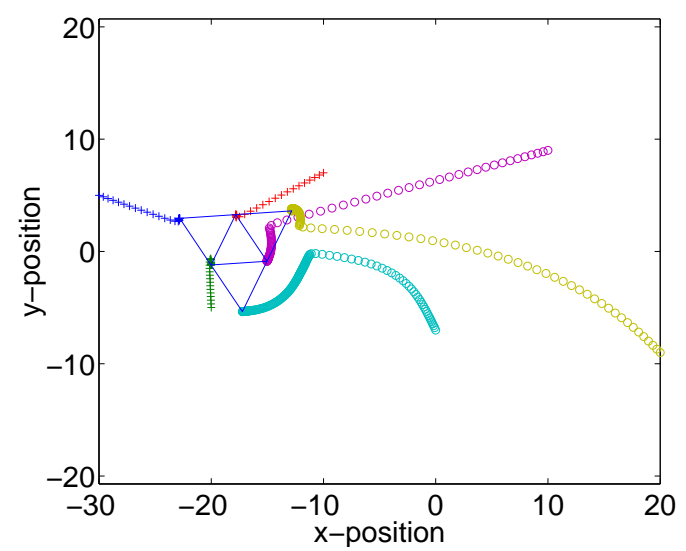

(a)

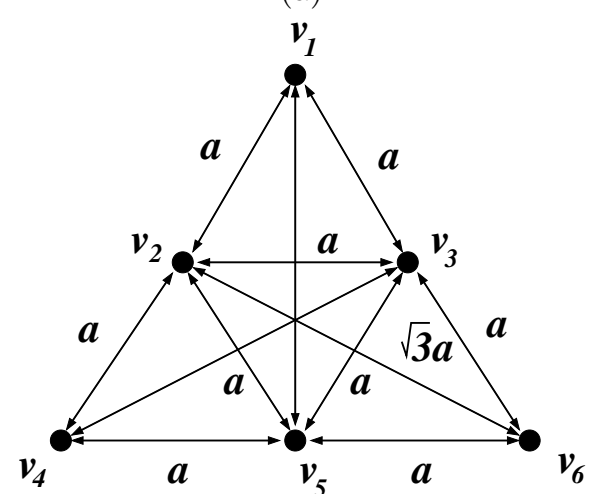

(b)

Fig. 3. Formation stabilization for 6 vehicles: (a) $q_{1}, \ldots, q_{6}$ and (b) formation graph $\mathcal{G}$. 